\title{
Molecules at the Interface of an HVC and a High-z HI Filament
}

U. Mebold, U. Herbstmeier, P.W.M. Kalberla, I. Souvatzis Radioastronomisches Institut der Universität Bonn Auf dem Hügel 71, D 5300 Bonn 1, F.R.G.

\section{Introduction}

The Draco nebula, the faint reflection nebulosity LBN $406 / 412 / 415$ (Lynds 1965 ) at $1 \approx 91^{\circ}, b \approx 38^{\circ}, z \geq 500$ pc (Goerigk and Mebold, 1986), contains delicate filaments at its low-l, low-b (SW) boundary (Goerigk et al. 1983, Mebold et al. 1985). The density in the boundary is characterized by the presence of molecules like co and $\mathrm{H}_{2} \mathrm{CO}$ (Mebold et al. 1985) and $\mathrm{NH}_{3}$ (Mebold et al. 1987). Johnson (1986) found an excess of unidentified IRAS point sources in the area of the Draco nebula, a large fraction of which is located close to the sw boundary.

The velocity field at the boundary of the Draco nebula is characterized by a gradient of about $+5 \mathrm{kms}^{-1} / 5^{\prime}$ on top of a systemic velocity of $\mathrm{V}=-22 \mathrm{kms}^{-1}$ (velocities refer to the LSR). The direction of the gradient is towards the galactic position angle $G P A=230^{\circ}$ measured from galactic north $(N)$ through the direction of increasing galactic longitude (E). The gradient along most of this boundary was dexived from HI observations made with the 100-m telescope (Goerigk et al. 1983). An association of the Draco nebula, in particular its SW boundary, with HI high velocity clouds (HVCs) has been discussed by Mebold et al. (1985) in the context of the origin of one of the filaments visible at prints of the Palomar observatory sky survey (POSS) by Rohlfs et al. $(1989 a)$.

In the present paper we present a complete map of the co distribution in the Draco nebula, review the evidence for an interaction of HVCs with the Draco nebula and the dust and HI cloud IVC $86.0+38.5-44$ located close to the Draco nebula. Finally we will discuss the origin of the molecules and the dust in these two nebulae in relation to their possible origin.

\section{Data and Results}

Fig.1 shows the $100 \mu \mathrm{m}$ emission of the Draco nebula (cf. Johnson 1.c.) observed by IRAS. The distribution of the integrated ${ }^{12} \mathrm{CO}$ line, WCO, is presented in Fig.2. The CO data were obtained with the 3-m KOSMA mm telescope at Gornergrat. The beam size is 3.9 arcmin, the velocity resolution is $\delta \mathrm{V}=0.08 \mathrm{kms}^{-1}$. The intensity scale is calibrated in $K\left(T_{R} *\right.$ ) and the $3 \mathrm{rms}$ temperature uncertainty is $\delta T=0.8 \mathrm{~K}$. More details of the data will be published elsewhere.

We see that the Co molecules are observed nearly exclusively at the low-l, low-b (SW) boundary of the Draco nebula. Comparing the WCO distribution with that of the $100 \mu \mathrm{m}$ emission, we find that the 
molecules are concentrated even more than the dust at a rather narrow strip along the fore-mentioned boundary. The intensity of the $100 \mu \mathrm{m}$ emission drops off very steeply to the $\mathrm{SW}$ and rather slowly to the NE of the nebula. This head-tail morphology is also present - but much less pronounced - in the wco-distribution.

The head-tail morphology of the dust- and the wco-distribution, the velocity gradient at the SW side of the HI distribution ( $s$. section 1) and the filamentary morphology of the dust boundary at the POSS suggest that the Draco nebula is moving towards the $\mathrm{SW}$ (galactic position angle $G P A=225^{\circ}$ ) and that shock fronts and instabilities exist at this boundary.

We have therefore studied the HI and the CO emission of this boundary with high angular resolution using the WSRT and VLA aperture synthesis telescopes for the HI- and the Onsala 20-m and the Kitt Peak 12-m single dish telescopes for the co-observations. Reports about these observations have been published (Rohlfs et al. 1989a) or will be published (Rohlfs et al. 1989b). Here we will discuss high resolution HI results relating to the velocity gradient and the narrow dust filaments at the sw boundary of the nebula and review high resolution co results relating to two suspected areas of interaction between HVCs and molecular gas.

Fig. 3 shows the velocity gradient observed perpendicular to the Sw-boundary in one of its undisturbed parts. The location is indicated by a heavy line in Fig.5. The gradient increases from a value $\approx 0.0 \mathrm{kms}^{-1}$ arcmin-1 inside the nebula monotoneously to a maximum value of $\approx+1.5 \mathrm{kms}^{-1} \mathrm{arcmin}^{-1}$ at the outer edge of the boundary. We have compared the location of that edge to that of the dust visible at the poss. We find that the edge of the dust distribution (indicated by the vertical line in Fig.3) is coincident within the positional accuracy of our VLA $21-\mathrm{cm}$ line data ( $\approx 30$ arcsec) with the location of the positive most HI gas at $\mathrm{V} \approx-12 \mathrm{kms}^{-1}$.

Fig. 4 shows a schematic presentation of the results found by Rohlfs et al. (1989a) for the dust filament which we will call DRA91.3+37.0-22. The frame of Fig.4 is indicated by its four corners in Fig.1. Rohlfs et al. suggest a model for the evolution of the filament during the last $10^{6} \mathrm{yr}$. At about that time an HVC consisting of a dense core $\left(\mathrm{n}_{H} \approx 100 \mathrm{~cm}^{-3}\right)$ and a less dense halo $\left(\mathrm{n}_{\mathrm{H}} \approx 0.6 \mathrm{~cm}^{-3}\right)$ which was located close to the upper left hand corner outside Fig.4, was moving along the direction of the vectors in that Figure and had a radial velocity of $V=-180 \mathrm{kms}^{-1}$. It started colliding with the boundary layer of the Draco nebula $\left(\mathrm{n}_{\mathrm{H}} \approx 10^{3} \mathrm{~cm}^{-3}\right)$ which had a radial velocity of $-18 \mathrm{kms}^{-1}$. During the inelastic collision a cloud, called the molecular clump in Fig.4, was formed from the combined mass of the HVC-core and the boundary layer. After the collision the clump left behind a turbulent wake which we see now as the filament connecting the clump and the Draco nebula. The presence of the wake indicates a large mass loss rate. Hence the clump appears to be a rather transient phenomenon. This conclusion is supported by the fact that the gravitational potential of the clump is not adequate to bind it. The authors can not give an estimate of the density of the medium into which the clump is moving now. 


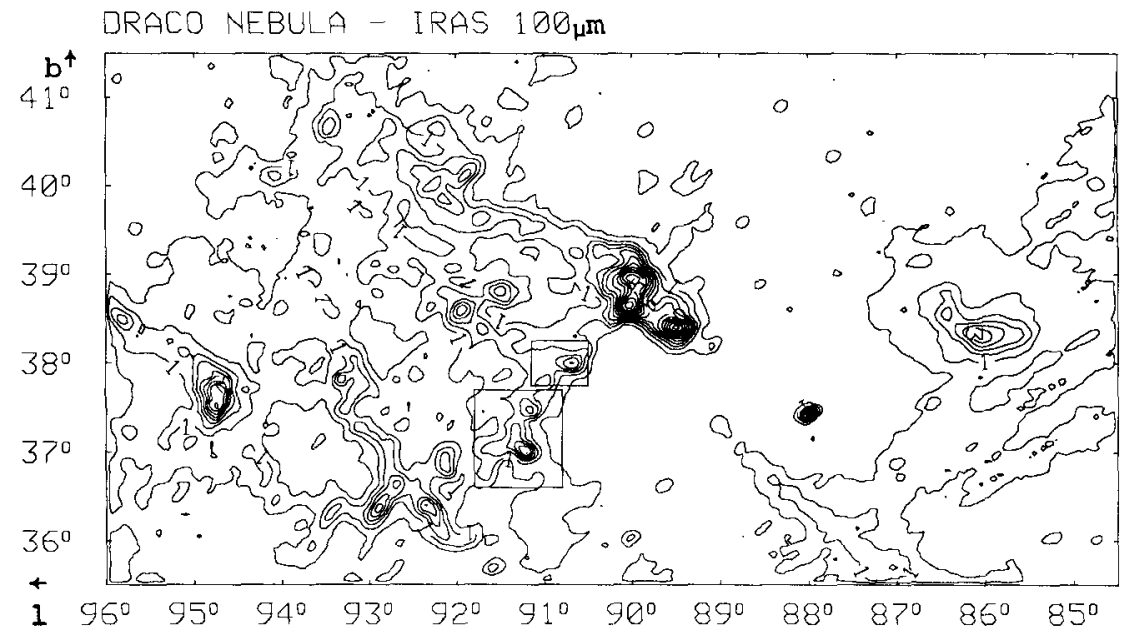

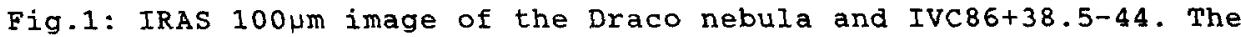
lowest contour is at $0.5 \mathrm{MJy} / \mathrm{sr}$ and the contour intervals are 0.5 MJy/sr. The frames of Figures 4 and 5 are indicated by rectangles.

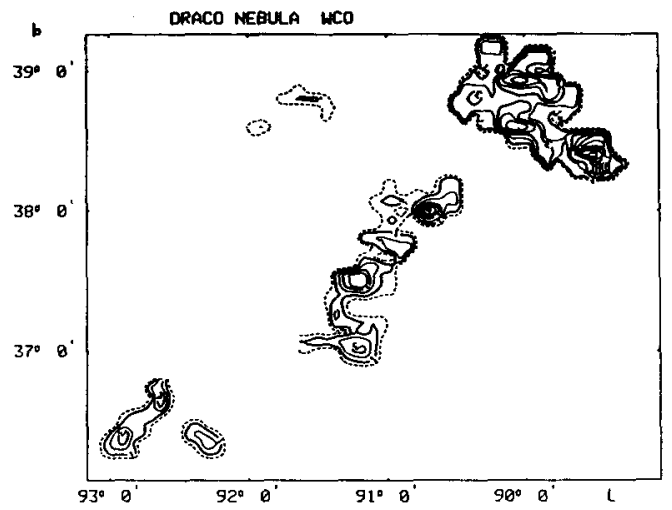

Fig.2: The ${ }^{12} \mathrm{CO}$ line integral of the Draco nebula observed with the KOSMA telescope and the mm-telescope at Bordeaux. The dashed contour corresponds to $0.5 \mathrm{kkm} / \mathrm{s}$, the first full contour is at $1 \mathrm{kkm} / \mathrm{s}$. The contour interval is $1 \mathrm{kkm} / \mathrm{s}$.

Fig.3: Position-velocity plot of HI brightness temperatures along the axis indicated in Figure 5. The lowest contour is at $2 \mathrm{~K}$ (Fig. $3 \mathrm{a}$ ) and $1 \mathrm{~K}$ (Fig. $3 \mathrm{~b}$ ) and the contour interval is $0.8 \mathrm{~K}$. The mark indicates the position of the dust edge at the poss plates.

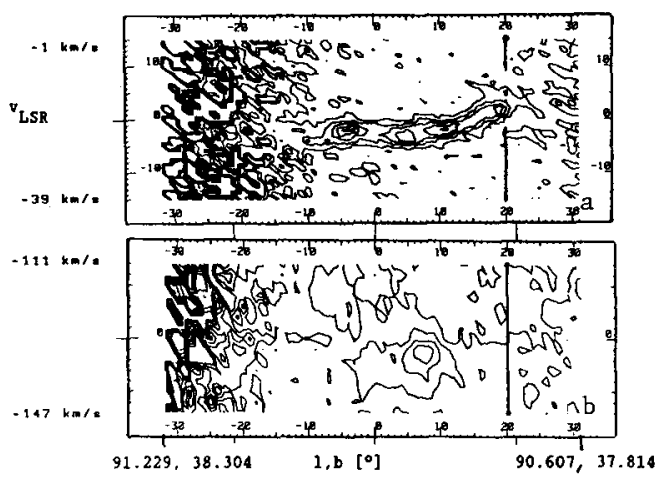


The quoted densities were derived from line intensities of $12 \mathrm{CO}$, $13 \mathrm{CO}$ and $\mathrm{H}_{2} \mathrm{CO}$ molecules and standard factors for the conversion of the corresponding molecular column densities into column densities of molecular hydrogen or hydrogen nuclei. It is likely that these conversion factors give too large densities (s. section 3 ).

Fig. 5 shows a schematic presentation of the results found by Rohlfs et al. (1989b) for the complex of filaments which we call DRA $90.7+38.0-20$. The frame of $\mathrm{Fig.5}$ is indicated by its four corners in Fig.1. The model proposed for the features in Fig.5 is similar to that just described with the main difference beeing that the collision of the boundary layer of the Draco nebula with HVCs, in particular the dense cores like HVC90.7+38.0-126, is much less evolved. The HVC clumps at velocities negative of $-133 \mathrm{kms}^{-1}$ are probably unaffected by the interaction with the Draco nebula and therefore indicate the initial velocity of the HVCs. The clump at $\mathrm{V}=-126 \mathrm{kms}^{-1}$ appears to be running into a molecular cloud at $\mathrm{V}=-$ $24 \mathrm{kms}^{-1}$ associated with the Draco nebula. This cloud has been accelerated towards the observer by $\approx 5 \mathrm{kms}^{-1}$. At the same time the HVC clump appears to have been decellerated by $\approx 10 \mathrm{kms}^{-1}$ compared to the velocity of the undisturbed HVC clumps. So, in the case presented in Fig.5 the collision has just started, while in Fig. 4 it apparently has already come to an end.

Fig. 6 shows the results of $21-\mathrm{cm}$ line observations with the $100-\mathrm{m}$ telescope of the third cloud which we believe to be the product of a collision of an HVC and gas at low velocities (Herbstmeier 1989). It is called IVC86+38.5-44, is located close to the Draco nebula and shows strong $100 \mu \mathrm{m}$ emission (see Fig.1). The cloud has also been observed with the KOSMA telescope for ${ }^{12}$ CO lines. No lines were detected to a limit of $T_{R}{ }^{*} \approx 0.4 \mathrm{~K}$. We see the cloud at the SW end of an HVC filament. This HVC filament appears as an extension of the head-tail elongation of IVC86+38.5-44. It can also be traced for $\approx 5^{0}$ along a faint dust tail starting at the core of the IVC and extending up to $1 \approx 90^{\circ}, b \approx 44^{\circ}$ (Herbstmeier 1989). The head-tail structure of the IVC and the HVC may be regarded as evidence for an interaction of the IVC with the surrounding medium. Such an interaction is also suggested by rather wide $21-\mathrm{cm}$ lines just at the SW edge of IVC86+38.5-44. The rather large dust colour temperature $(\approx 25 \mathrm{~K})$ derived from the $60 \mu \mathrm{m}$ and $100 \mu \mathrm{m}$ IRAS emission may or be not related to a possible interaction.

\section{Discussion and Conclusion}

An interesting aspect of these high-z dust and molecular clouds is their internal pressure. While Wakker (this volume) gives rather low values for the pressure in his HVCs, Souvatzis (1989) finds rather large values $\left(n * T \approx 10^{5} \mathrm{Kcm}^{-3}\right)$ for the HVC clumps in Fig.5. Here $n * T$ has been estimated from the HI column density the linear size and the observed velocity dispersion. The $n \star T$ values for the HI gas in the associated features of the Draco nebula are close to $10^{4} \mathrm{Kkms}^{-1}$, i.e. a factor of $\approx 10$ smaller (Souvatzis 1989). If the partial pressure of the molecular constituent of the Draco nebula is added, the total pressure may be close to that in the associated HVCs. Although it is difficult to compare the $n * T$ values for our high-z clouds with those $\left(\approx 10^{4} \mathrm{Kkms}^{-1}\right)$ derived for galactic plane clouds, the former values are probably clearly 


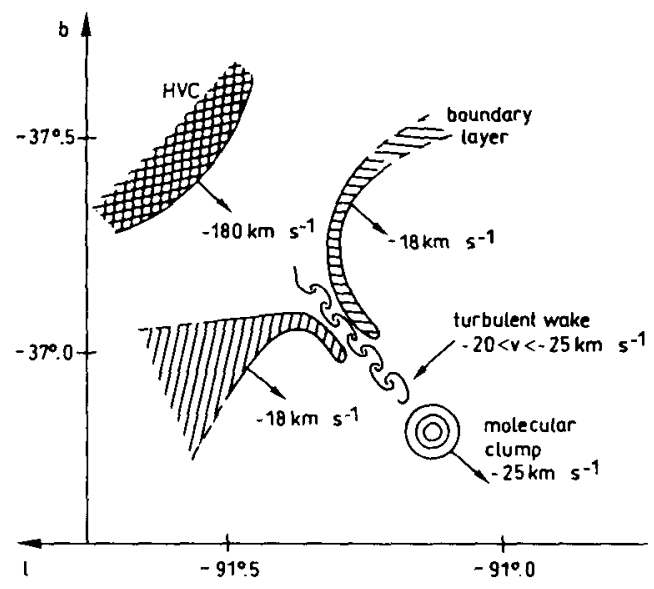

Fig.5: Sketch of the collision scenario around HVC90.7+38.0126 indicated by " $\%$ ". The velocities are relative to the ISR. For details see text. The location of the position velocity plot in Fig. 3 is indicated by "XV".



Fig.4: Sketch of the collision scenario DRA91.3+37.0-22. The velocities are relative to the LSR. For details see text.

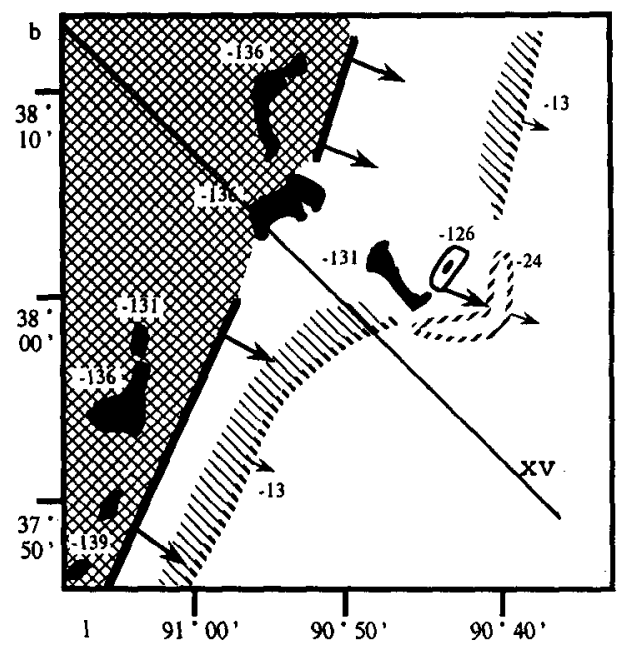

Fig.6: HI column density distribution of IVC $86+38.5-44$ (full lines) and the HVC filament (dashed lines). The contours are labelled in $10^{19} \mathrm{~cm}^{-2}$. 
larger: For similar values of the temperature $\left(10^{3} \mathrm{~K}\right)$ the densities in our HVC clumps are probably larger by a factor of $\approx 10$.

The rather large pressures found in the HVCs and the boundary layer of the Draco nebula is additional evidence for non-steadystate conditions, e.g. some kind of collisional interfaces, in this area (cf F.Kahn, this volume). From this evidence and the results given in section 2 we obviously can identify two different kinds of collision fronts:

1 . There is the high velocity $\left(\delta \mathrm{V} \approx 100 \mathrm{kms}^{-1}\right)$ collision between the HVCs and the Draco nebula. This kind of collision is observed in the area displayed in Fig.5, has occured - according to the model of Rohlfs et al. (1989a) - some $10^{5} \mathrm{yr}$ ago in the area displayed in Fig. 4 and has possibly produced the cloud IVC86+38.5-44 (Fig.6). 2. The other kind of collision is the low velocity $\left(\delta \mathrm{V} \approx 20 \mathrm{kms}^{-1}\right)$ collision between the Draco nebula and the gas at zero velocties, i.e. the medium at the SW side of the Draco nebula.

We propose to interpret the Draco nebula as well as IVC86+38.5-44 as the results of collisions between HVCs and an HI filament which has a velocity close to zero and is reaching out of the galactic plane to $b \approx 40^{\circ}$ (Heiles 1984). We hypothesize that it extends to an elevation of $z \geq 500 \mathrm{pc}$.

The cloud IVC $86+38.5-44$, as the least complicated structure of those discussed here, may be considered as the region between the down-stream and the up-stream shocks which have developped after a collision of a HVC at a velocity of $\mathrm{V}_{\mathrm{HV}} \mathrm{C}=-110 \mathrm{kms}^{-1}$ versus the zero-velocity filament. Here the direction of the motion is assumed to be parallel to the tail in Fig.6. Using the relation

$\mathrm{V}_{\mathrm{CD}}=\mathrm{V}_{\mathrm{H} V \mathrm{C}} /\left(1+\left(\mathrm{n}_{\mathrm{H}} \mathrm{C}_{\mathrm{C}} / \mathrm{n}_{0}\right)-1 / 2\right)$

for the velocity of the contact discontinuity, $V_{C D}$, as a function of $V_{H V C}$ and the densities of the HVC, $n_{H V C}$, and the zero-velocity filament, no, we can reproduce the velocity of our IVC, VIvc=$44 \mathrm{kms}^{-1}$, if we adopt a density contrast of $\mathrm{n}_{\mathrm{ac}} / \mathrm{n}_{0}=0.3$ and identify $V_{I} v_{c}$ with $V_{C D}$. The adopted density contrast is realistic for the kind of high-z HI filament considered here. The latter condition may be fullfilled if the material cools rapidly behind the shock. In this picture the SW edge of the IVC is identified with the down-stream shock, its NE end is identified with the upstream shock and cooled material is the HI gas which we observed.

A similar explanation may be given for the Draco nebula. We can reconcile its velocity of $-22 \mathrm{kms}^{-1}$ by a collision of HVCs with velocities between -180 and $-130 \mathrm{kms}^{-1}$ if the density contrast is much larger than that in the case of IVC86.0+38.5-44: $\mathrm{n}_{\mathrm{H}} \mathrm{c} / \mathrm{n}_{0}=$ 0.02 to 0.04 , respectively. Here the relative velocity of the upstream shock is between 160 and $100 \mathrm{kms}^{-1}$ while that of the downstream shock is only close to $22 \mathrm{kms}^{-1}$. It is suggestive to identify the up-stream shock with the SW boundaries of the HVCs in Fig.4 and Fig.5. The down-stream shock is then at the sw edge of the Draco nebula, in the region were the velocity gradient displayed in Fig. 3 - is found. As predicted by our model, a velocity gradient similar to that in Fig. 3 is found for most of the SW boundary of the Draco nebula. 
In this model the up-stream shock is a fast shock which probably dissociates molecules and possibly even destroys dust particles. The down-stream shock is a slow shock which allows for rapid cooling and the building up of the molecules which we have observed in the post-shock region of the Draco nebula. The $100 \mathrm{um}$ emission of the dust, which is seen in this region too, has been swept up behind the slow shock and in front of the contact discontinuity from the material into which the shock is moving. The contrast of the gas and dust density behind the slow shock and that in the general field in front of the slow shock may therefore be used as an indicator of the length of the path of the HVCs through our Galaxy.

Summing up, the proposed collision of HVCs versus a high-z HI filament qualitatively explains most of the observed features of the Draco nebula and IVC $86+38.5-44$. However, a large number of details have to be worked out before this model is completely acceptable. In this context and finally in this paper, we will discuss the conversion of molecular line intensities, in particular WCO, into column densities of molecular hydrogen or hydrogen nuclei. An analysis of the data of the Draco nebula indicates that molecules, in particular $c 0$, are overabundant compared to typical clouds in the galactic plane (Herbstmeier et al. 1989). Similar results were also found for Cirrus clouds (Heithausen and Mebold 1989). An explanation of the apparently large molecular abundances may either be related to the reduced depletions of the heavy elements found towards stars in high galactic latitudes or related to shocks - similar to those discussed in the present paper - which may produce an enhancement of molecules in the Cirrus (cf e.g. the Discussion in Heithausen and Mebold 1989). Although this problem does not affect the rather qualitative interpretation given above, it is of major importance, if these or similar clouds are used to study phenomena like shock fronts or are used as probes for the astrophysical conditions in the halo of our Galaxy

\section{References}

Goerigk, W., Mebold, U., Reif, K., Kalberla, P.M.W., Velden. L.: 1983, Astron. Astrophys. 120, 63

Goerigk, W., Mebold,U.,:1986, Astron. Astrophys. 162, 279

Heiles, C.: 1984, Astrophys. J. Suppl. 55, 585

Heithausen, A., Mebold, U.: 1989, Astron. Astrophys. 214, 347

Herbstmeier, U., Rohlfs, R., Mebold, U.: 1989, in: "The Physics and Chemistry of Interstellar Molecular Clouds", ed.

G. Winnewisser and J.T. Armstrong, Springer Verlag.

Herbstmeier, U.:1989, in prep.

Johnson, H.M.: 1986, Astrophys. J., 309, 321

Lynds, B.T.: 1965, Astrophys. J. Suppl. , 12, 163

Mebold, U., Cernicharo, J., Velden, L., Reif, K., Crezelius, C., Goerigk, W.: 1985, Astron. Astrophys. 151, 427

Mebold, U., Heithausen, A., Reif, K.: 1987, Astron. Astrophys. 180,213

Rohlfs,R., Herbstmeier, U., Mebold, U., Winnberg, A.: 1989a, Astron. Astrophys. 211, 402

Rohlfs,R., Herbstmeier,U., Mebold,U., Fink, U.:1989b, Astron. Astrophys, subm.

Souvatzis,I.1989: Diploma Thesis, University of Bonn 


\section{Discussion:}

WAKKER: Do you expect that the high velocity gas becomes ionized at the interface between low and high-velocity gas that you claim to see. If so, what about $\mathrm{H} \alpha$ emission from that region? Is that expected there?

MEBOLD: We do expect ionized gas at this interface and I know that G. Münch has tried to find it. I am not sure about his results, but obviously any possible emission is not strong. 